\title{
EDITORIAL
}

\section{Lipopolysaccharide and the lung: a story of love and hate}

\author{
R. Bals
}

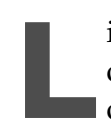
ipopolysaccharide (LPS) is a major component of the outer membrane of Gram-negative bacteria. LPS is one of the most potent microbial inducers of inflammation and is responsible for many of the deleterious effects of pulmonary infections. In contrast, surfaces of the body are constantly exposed to small amounts of LPS. This interaction appears to stimulate more beneficial, protective mechanisms. Overall, the interactions between LPS and the body are important; however, factors that determine the nature of this response are not known.

LPS is a prototypical pathogen associated molecular pattern (PAMP), a term that was introduced by JANEWAY and MeDZHITOV [1], which describes structurally conserved molecules of microorganisms. PAMPs bind to receptors of the innate immune system, consequently called pattern recognition receptors (PRRs). In 1996, the Drosophila protein Toll was shown to be required for flies to mount an effective immune response against the fungus Aspergillus fumigatus. In 1998, toll like receptor (TLR) 4 was positionally identified as the LPS receptor and found to be required for mice to effectively respond to Gram-negative bacteria. These findings have guided the field of (innate) immunity over the last few years. Thirteen mammalian TLR analogues have been identified and functionally linked to various processes, ranging from inflammation and infection, to arthrosclerosis and sterile inflammation [2].

In the lung, TLR4 is expressed by several different cell types, including professional host defence (dendritic cells, macrophages) and structural (endothelial and epithelial cells) cells. Animal models and patient studies have proved that TLR4 contributes significantly to the response to inhaled LPS [3, 4]. It is obvious that high levels of LPS initiate a host defence reaction with subsequent activation of the adaptive immune system. Interestingly, low levels of LPS also appear to influence the biology of the lungs. The respiratory tract is constantly exposed to LPS contained in cigarettes, bedcovers or elsewhere. Epidemiological evidence suggests that the level of inhaled LPS during early childhood determines susceptibility to allergic airway disease [5]. The presence of bacterial PAMPs such as LPS and its recognition by epithelial cells is important for tissue homeostasis in the gastrointestinal tract [6]. LPS obviously serves not only as an exogenous danger signal, but

CORRESPONDENCE: R. Bals, Dept of Internal Medicine, Division of Pulmonary Medicine, Hospital of the University of Marburg, Baldingerstrasse, 35043 Marburg, Germany. Fax: 4964212868987. E-mail: bals@mailer.uni-marburg.de also as a beneficial exogenous compound modulating processes such as adaptive immunity or tissue repair.

Taken together, the interaction of LPS and the lung is important and complex. Experimental information is needed from a number of different levels. This issue of the European Respiratory Journal contains two papers that characterise the interaction of LPS and the lung. The first study addresses the mechanisms that are involved in the pulmonary response to LPS exposure. The study of EUTAMENE et al. [7] investigates the role of the myosin light chain kinase (MLCK) in the response to LPS applied to the respiratory tract. The authors engage a rat animal model and further clarify cellular mechanisms using tissue culture techniques. MLCK activity has mainly been linked to the biology of the airway smooth muscle or endothelial cells [8], but also to epithelial permeability [9]. The scientists use the specific MLCK inhibitor ML-7. The data from the animal experiments show that ML-7 inhibits the neutrophilic inflammation caused by LPS. In vitro, phosphorylation of MLCK after LPS exposure was inhibited by ML-7. These data provide evidence that the regulation of tight junctions in the lung is important for the inflammatory reaction and the influx of inflammatory cells. For many diseases, such as classical inflammatory diseases like asthma or COPD, this issue hasn't been addressed. It would be interesting to establish whether classical recognition mechanisms or signalling pathways are involved in the activation of MLCK. There is evidence that besides the CD14-TLR4 complex other membrane proteins could be involved in the recognition of LPS.

The second study applied a human model of LPS exposure. There are only a small number of studies that have investigated the response of healthy volunteers to exposure with LPS. On the one hand, this type of study is very important because it is the ultimate goal in the understanding of the processes in the human lung. On the other hand, these studies are difficult to interpret because they are necessarily limited in the number of probands, and the effect of LPS certainly depends on dose, age of the probands, route of administration, duration of exposure and many other factors. The study of ROOS-ENGSTRAND et al. [10] investigates the response of the lung to exposure with inhaled LPS in healthy volunteers. The authors analyse signalling cascades and focus on the airway epithelium. They found a significantly increased expression of p38 MAPKs, a signalling molecule with a central position in inflammatory reactions. IL-8 and other cytokines did not show a significant change. Based on the material obtained from patients or probands, the methods applied for characterisation are limited. The present study engages a sophisticated 
approach to investigate intracellular signalling. As many signalling molecules are activated by phosphorylation or other post-transcriptional modifications, the appropriate tools to detect these changes have to be used. In the present study, the authors use antibodies directed to the phosphorylated forms of the signalling molecules and engage an elaborate approach of quantification. Nevertheless, the methodology of morphological studies to address signalling processes is challenging.

Both studies provide important data from different levels in the interaction between LPS and the lung. The complexity of the interaction between LPS and the body is reflected by the multitude of signalling pathways that have been described to be activated by LPS. The TLR/interleukin-1 receptor pathway is certainly one of the most prominent and is localised very proximally. Downstream signalling involves mitogenactivated protein kinases (MAPK), such as p38, described in one of the studies in this issue. The MAPK pathways transduce a large variety of external signals, leading to a wide range of cellular responses, including growth, differentiation, inflammation and apoptosis. Many questions have to be addressed to further clarify the relationship between LPS and the lungs in order to apply this knowledge in medicine.

1) What are the key cells that regulate the response to LPS? It is obvious that airway and parenchymal epithelial cells contribute significantly to the inflammatory response, but are they alone capable of recognising LPS? Experimental evidence suggests that macrophages are important in the initial recognition of LPS and then trigger epithelial cells.

2) Is the application of LPS equivalent to exposure to other PAMPs or even viable bacteria? The innate immune system is equipped with many PRRs and is probably capable of discriminating between different classes of microorganisms.

3) Is the dose and the timing (acute versus chronic) important? Almost certainly, yes. It has also been shown that different levels of LPS cause inhibition or stimulation of T-helper 2weighted responses [11].

4) Is the outcome of LPS exposure always detrimental or can it, in some situations, be beneficial? It would be surprising if the body was unable to somehow deal with small amounts of LPS. This exposure was constantly present during the evolution of Homo sapiens. The task is now to clarify whether this low level exposure has a role in pulmonary diseases.

5) What are the mechanisms of LPS recognition? Is it all based on TLRs or are other mechanisms involved? Surprisingly, neither article apply the TLR concept to their experimental set-up.

6) Can signalling pathways of the innate immune system or certain components be targeted to protect against the harmful consequences of LPS recognition, leaving host defence mechanisms intact? A detailed understanding of the various signalling pathways and their balance would be necessary [12].

The interaction between lipopolysaccharide and the lung is important for health and disease. Many more data points have to be filled in the matrix to further understand this complex interaction.

\section{REFERENCES}

1 Janeway CA Jr, Medzhitov R. Innate immune recognition. Annu Rev Immunol 2002; 20: 197-216.

2 Beutler B. Inferences, questions and possibilities in Tolllike receptor signalling. Nature 2004; 430: 257-263.

3 Hawn TR, Verbon A, Janer M, et al. Toll-like receptor 4 polymorphisms are associated with resistance to Legionnaires' disease. Proc Natl Acad Sci USA 2005; 102: 2487-2489.

4 Arbour NC, Lorenz E, Schutte BC, et al. TLR4 mutations are associated with endotoxin hyporesponsiveness in humans. Nat Genet 2000; 25: 187-191.

5 Braun-Fahrlander C, Riedler J, Herz U, et al. Environmental exposure to endotoxin and its relation to asthma in school-age children. N Engl J Med 2002; 347: 869-877.

6 Rakoff-Nahoum S, Paglino J, Eslami-Varzaneh F, Edberg S, Medzhitov R. Recognition of commensal microflora by toll-like receptors is required for intestinal homeostasis Cell 2004; 118: 229-241.

7 Eutamene H, Theodorou V, Schmidlin F, et al. LPS-induced lung inflammation is linked to increased epithelial permeability: role of MLCK. Eur Respir J 2005; 25: 789-796.

8 Matsumura F, Totsukawa G, Yamakita Y, Yamashiro S. Role of myosin light chain phosphorylation in the regulation of cytokinesis. Cell Struct Funct 2001; 26: 639-644.

9 Ma TY, Boivin MA, Ye D, Pedram A, Said HM. Mechanism of TNF- $\alpha$ modulation of Caco-2 intestinal epithelial tight junction barrier: role of myosin light-chain kinase protein expression. Am J Physiol Gastrointest Liver Physiol 2005; 288: G422-G430.

10 Roos-Engstrand E, Wallin A, Bucht A, Pourazar J, Sandström T, Blomberg A. Increased expression of p38 MAPK in human bronchial epithelium after lipopolysaccharide exposure. Eur Respir J 2005; 25: 797-803.

11 Eisenbarth SC, Piggott DA, Huleatt JW, et al. Lipopolysaccharide-enhanced, toll-like receptor 4-dependent T helper cell type 2 responses to inhaled antigen. J Exp Med 2002; 196: 1645-1651.

12 Ulevitch RJ. Therapeutics targeting the innate immune system. Nat Rev Immunol 2004; 4: 512-520. 